\title{
Controlling the Reaction Process in Operando STEM by Pixel Sub-Sampling
}

\author{
B. Layla Mehdi ${ }^{1}$, Andrew Stevens ${ }^{2}$, Libor Kovarik ${ }^{3}$, Andrey Liyu ${ }^{3}$, Bryan Stanfill ${ }^{2}$, Sarah Reehl ${ }^{2}$, \\ Lisa Bramer ${ }^{2}$, Nigel D. Browning ${ }^{1,4}$ \\ ${ }^{1}$ Physical and Computational Science Directorate, PNNL, Richland, WA 99352, USA \\ ${ }^{2}$ National Security Directorate, PNNL, Richland, WA 99352, USA \\ ${ }^{3}$ Environmental Molecular Sciences Laboratory, PNNL, Richland, WA 99352, USA \\ ${ }^{4}$ Materials Science and Engineering, University of Washington, Seattle, WA 98195, USA
}

Recently there has been an increase in the number of experiments making use of either in-situ gas or liquid stages, or using dedicated environmental (scanning) transmission electron microscopes (S/TEM) to study dynamic materials processes. While in-situ observations have traditionally been performed in TEM mode, allowing the intrinsic increase in temporal resolution of the projection image to be utilized, there are a number of key advantages of using the STEM imaging mode for these experiments. Namely, the same physics that makes high angle annular dark field (HAADF) or Z-contrast imaging optimum for quantifying small metal/oxide catalyst particles on a support also makes it optimum for imaging particle dynamics in liquids (now the liquid is the background in the image rather than the support). In addition, the incoherence of the Z-contrast image (i.e. decreased sensitivity to thickness effects) makes it the ideal method to image through the $\sim 100-500 \mathrm{~nm}$ thick in-situ liquid cells that are typically used. Somewhat counter intuitively, the STEM imaging process is also optimized for controlling and reducing beam damage - the dose is controlled by the beam size and dwell time of the scan, while the probe only illuminates a small area, thereby reducing heating and depletion effects [1].

The use of the scanned beam to form images has another advantage in that it can readily make use of compressive sensing/in-painting approaches [2,3] to reduce the overall dose during the experiment and increase the acquisition speed [4,5]. The in-painting method is a mechanism by which a small sub-set of pixels can be acquired experimentally and then mathematical processes used to in-paint the missing information. Demonstration of this approach in practical acquisition of images [5] indicates that full reconstruction can be obtained for sub-sampling down to the $\sim 10 \%$ level. In addition to reconstructing the images, analytics to quantify the content of the images has also been shown to work for extremely low sampling rates [6]. Such results suggest that an extra degree of flexibility in performing in-situ liquid or gas experiments can be built in by changing the level of sampling, i.e changing the density of dosed pixels used to acquire the images.

Figure 1 shows the final fully sampled images obtained after in-situ liquid nucleation and growth experiments were performed on a $10 \mathrm{mM} \mathrm{AgNO}_{3}$ solution sampled in full mode (Fig 1A) and $12 \%$ subsampled mode (Fig. 1B). What can be clearly seen from these results that the sub-pixel sampling leads to different growth morphology of silver nanoparticles. In this presentation, a discussion on how subsampling affects the growth mode for this and other in-situ liquid experiments will be discussed in detail [7]. 


\section{References:}

[1] T. J. Woehl, K. L. Jungjohann, J. E. Evans, I. Arslan, W. D. Ristenpart, and N. D. Browning, Ultramic. 127, 53-63 (2013)

[2] E. J.Candès, J. Romberg, T. Tao, Inform. Theory IEEE Trans. 52, 489-509 (2006)

[3] D. L. Donoho, Inform. Theory IEEE Trans. 52, 1289-1306 (2006)

[4] A. Stevens, H. Yang. L. Carin, I. Arslan and N. D. Browning, Microscopy 63, 41-51 (2014)

[5] L. Kovarik, A. Stevens, A. Liyu, N. D. Browning, Applied Physics Letters 109, 164102 (2016)

[6] B. Stanfill, S. Reehl, D. Ries, M. Johnson, B. L. Mehdi, N. D. Browning, L. Bramer, submitted

[7] This work supported by the Chemical Imaging Initiative (CII) and the Analytics in Motion (AIM) Initiative, Laboratory Directed Research and Development (LDRD) Programs at Pacific Northwest National Laboratory (PNNL). PNNL is a multi-program national laboratory operated by Battelle for the U.S. Department of Energy (DOE) under Contract DE-AC05-76RL01830. A portion of the research was performed using the Environmental Molecular Sciences Laboratory (EMSL), a national scientific user facility sponsored by the Department of Energy's Office of Biological and Environmental Research and located at PNNL.
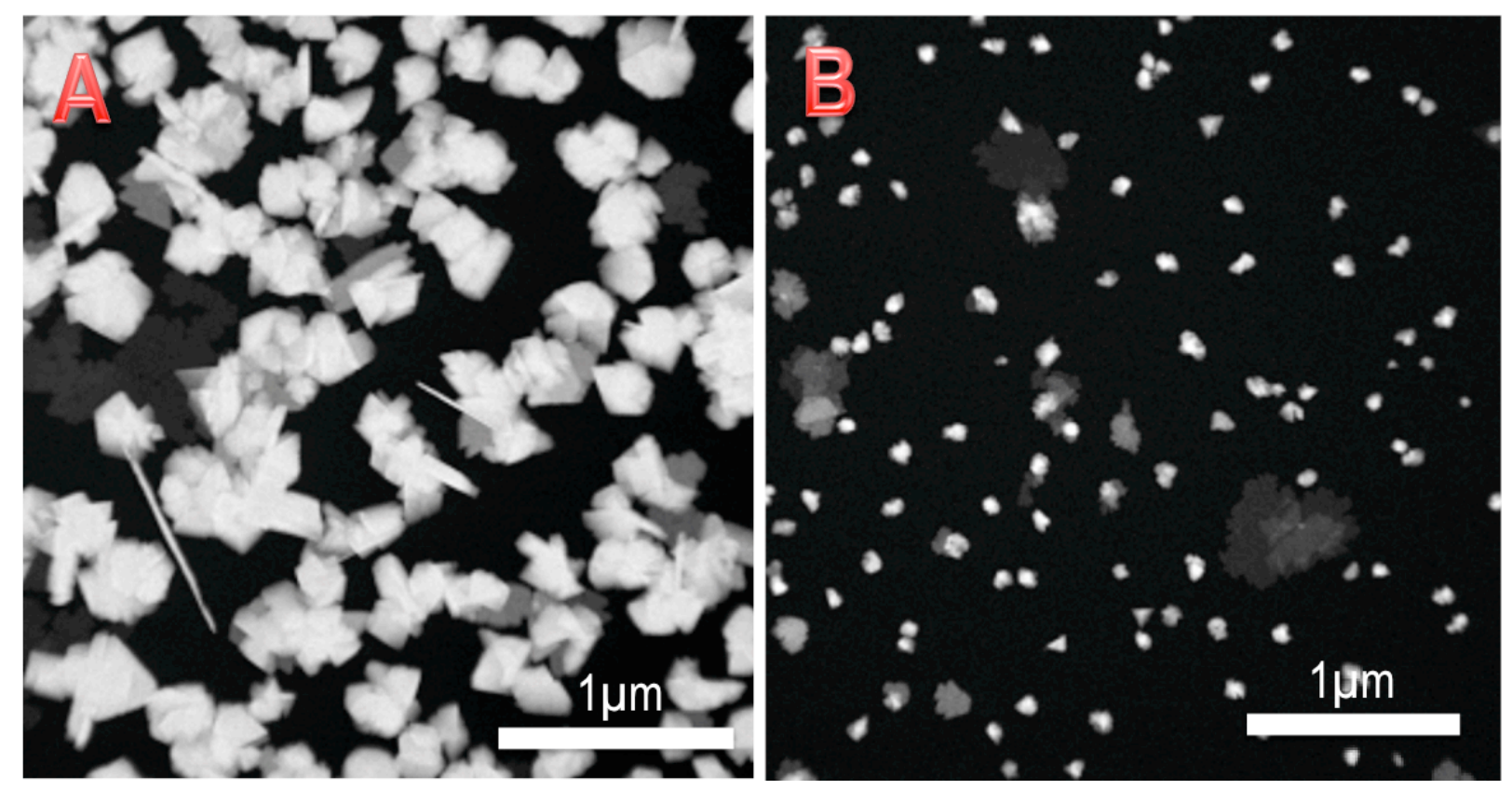

Figure 1. HAADF STEM image of Ag nanoparticles acquired in (A) full mode and (B) $12 \%$ subsampled mode. 